\title{
Jihadist expression in the scripts of the film "The Lion of the Desert"

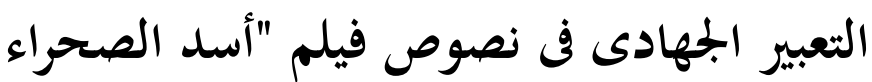

\section{Zaenal Masduqi}

\section{Zaenalmasduqi85@yahoo.com}

Dirasât al-Islamiyah wa al-'Arabiyah

Sunan Kalijaga State Islamic University Yogyakarta, Indonesia

\section{Sangidu}

sangidu@ugm.ac.id

Department of Western Asia Study, Faculty of Cultural Sciences

Gadjah Mada University, Indonesia

• Received: 01.03.2021 • Accepted: 30.04.2021 • Published online: 05.05.2021

Abstract: This study is intended to describe the character of Omar Mukhtar visualized in the film entitled 'Lion of the Desert' and to analyze jihad expressions in the form of statements or questions in the film. This research has used a qualitative approach using narrative analysis method. The subject of this research is the film "The Lion of the Desert" by Moustapha Akkad, while the object of research is the narrative and dialogue of the scenes in the film which are related to the expression of jihad in the film "The Lion of the Desert". The technique of collecting data is by doing documentation techniques, the whole data is described and interpreted so as to produce a descriptive data discussion. The character of Omar Al-Mukhtar is shown in the film as a figure of a believer who holds fast to his religious values, he is also a devout patriot who always increases the fighting spirit of the Libyan people to be free from the grip of the Italian state colonialism. There are seven expressions found in the film's narrative as expressions of jihad, either explicitly or implicitly.

Keywords: Jihad, Omar Mukhtar, the Desert Lion Movie, Narration

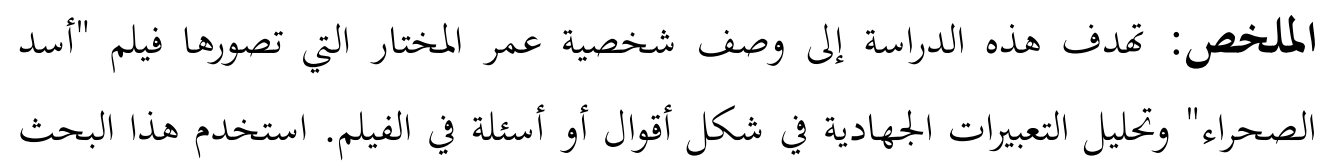




\section{Zaenal Masduqi}

المنهج النوعي باستخدام أسلوب التحليل السردي. موضوع هذا البحث فيلم "أسد الصحراء" لمصطفى العقاد ، أما موضوع البحث فهو سرد وحوار المشاهد في الفيلم والتي تتعلق بالتعبير عن الجهاد في فيلم "أسد الصحراء". الصحراء". تقنية جمع البيانات عن طريق القيام بتقنية التوثيق ، يتم وصف البيانات بالكامل وتفسيرها لإنتاج مناقشة بيانات وصفية. تظهر شخصية عمر المختار في الفيلم كشخصية مؤمن يتمسك بقيمه الدينية ، كما أنه وطني متدين يزيد من الروح القتالية للشعب اللبيي حتى يتحرر من قبضة الإيطاليين. احتلال الدولة. هناك سبعة تعبيرات

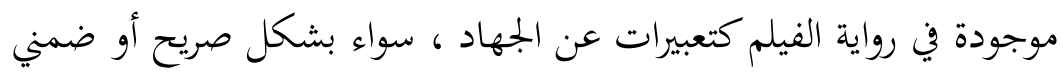
كلمات دلالية: الجهاد، عمر مختار، فيلم أسد الصحراء، السرد

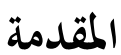

لا خلاف بين أهل العلم أن التعبير القرآني تعبير فريد في علوه و سموه وأنه أعلى كلام

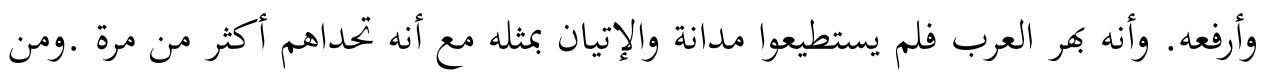

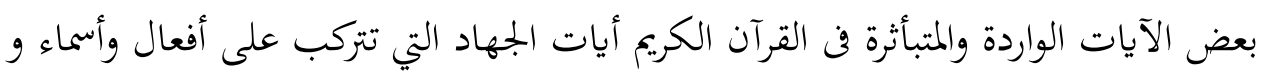
أساليب بذوق بلاغي وتاريخي وتعبير خاص تكون أساسا ومركزا ومثاليا لظهور الأساليب المتبعة ما بعدها. (Rosyid, Isbah, \& Assegaf, 2020) وأما التراكيب والأساليب الجهادية التى يقولها عمر المختار شيخ المجاهدين وإخواته في

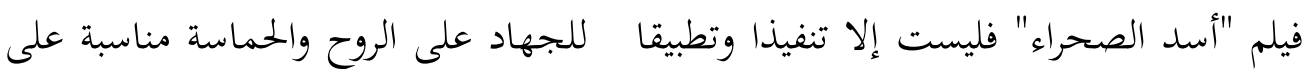
وقت وسبب وإقليم مخصوص. إنه بجرد حالة من حالات الحرب العديدة التى وقعت بين المسلمين ومن كان عدوهم الذي يحاربهم ويحتل بلادهم. وآيات الجهاد في القرآن تبقى صالحة الأبد مع تاريخ يعيد نفسه في أوقات وأماكن ومرتكبى مختلفين. Hourani, 1991; Pandawa, 2021) هناك العديد من الطرق التى استخدمها الناس لإيصال المعلومات السابقة والرسائل

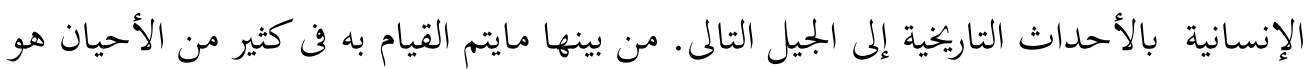
تأليف الكتب التاريخية وصناعة الأفلام الوثائقية. بكتين وسيلتين يمكن تدويم إيصال المعلومات بئن التاريخية السابقة حتى يعلمها كثيرمن الناس, يعرف هذا في علم التاريخ باسم استخدام دراسة 


\section{Zaenal Masduqi}

تاريخية الدرجة الثانية. من الواجب, صناعة الأفلام التاريخية كمثل فيلم أسد الصحراء تحتاج الكثير من المال والمهارة فن جميع المجال لإنجاحه. بالإضافة إلى استخلاص حقائق تاريخية كاملة, رغم أنه سيكون هناك خيال يجمل الفيلم لتكون جذابة يدافع الناس على مشاهدته وأخذ بـان الدروس النافعة للحياة من الفيلم.

\section{منهجية البحث}

استخدم هذا البحث المنهج النوعي باستخدام أسلوب التحليل السردي. يمكن استخدام طريقة لدراسة بنية الرسائل أو حول الوظائف المختلفة للغة (براغماتية) ، والتحليل السردي وفقًا لسوبر Sobur (2001) لدراسة بنية القصص من الروايات الخيالية (مثل الروايات والأفلام). السرد هو تمثيل للأحداث ، لذلك تم اختياره كأسلوب بحث لأن هذا التحليل السردي يرى النص على أنه قصة ، فيها حبكات ومشاهد وشخصيات وشخصيات.

موضوع هذا البحث فيلم "أسد الصحراء" لمصطفى العقاد ، أما موضوع البحث فهو سرد وحوار المشاهد في الفيلم والتي تتعلق بالتعبير عن الجهاد في فيلم "أسد الصحراء". الصحراء". تم استخدام تقنية جمع البيانات من قبل الباحثين في فحص محتوى الرسالة في فيلم "أسد الصحراء" من خلال تنفيذ تقنيات التوثيق ، وهي تقنية جمع البيانات عن طريق فحص الملاحظات أو الوثائق وكذلك المراقبة المباشرة لموضوع البحث في شكل قرص DVD من فيلم "أسد الصحراء". سيتم تحليل جميع البيانات التي تم الحصول عليها وبمعها بناءً على النظريات ذات الصلة. في النهاية ، سيتم وصف جميع البيانات وتفسيرها لإنتاج مناقشة بيانات وصفية.

\section{نتائج الدراسة وتحليلها}

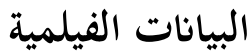

أخبرنا فيلم إذاعة الجهاد وتحرير البلاد بالموضوع "أسد الصحراء" شيخ المجاهدين عمر المختار أن الشخصية في هذه القصة حقيقية وأحداثها مبنية على الوقائع التاريخية. حكى هذاء لهيخ 
Zaenal Masduqi

الفيلم عن حركة بطل ليبيا عمر المختار. شجاعته الواثقة وخبرته الفائقة في الأمور السياسية

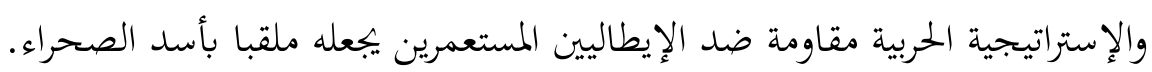

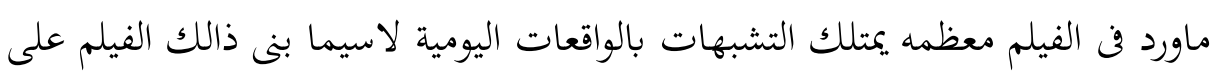

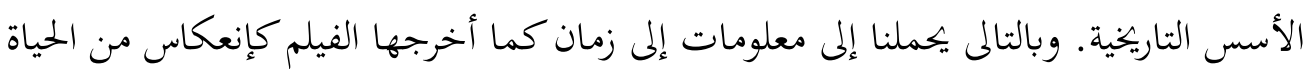
الحقيقية. كل تعبيرات الموجودة فن فيلم أصدرت على حالة مخصوصة مأخوذة من تحقيق الحياة

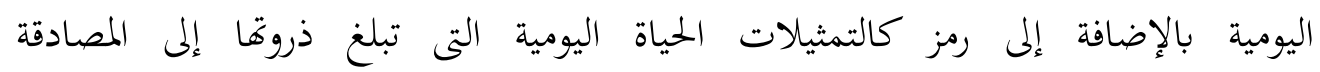
والتجنيس.(Rizki \& Golubović, 2020) هذا الفيلم الضخم الذى أنتجه المخرج السينمائي مصطفى العقاد، مسلم من حلب

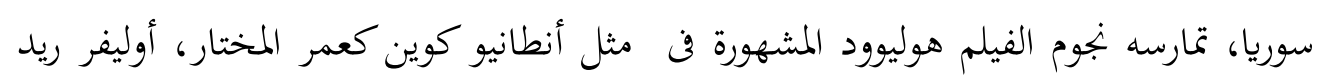
كجنرال رودلفو غراتسياني الولي لليبيا، ريد ستيغر كبنيطو موسوليني، وغير ذالك على حساب

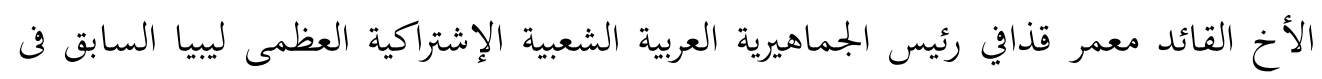

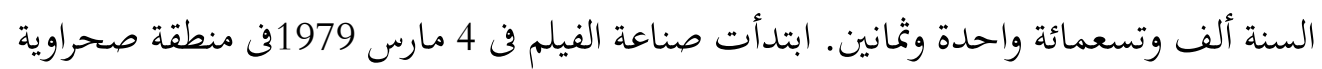

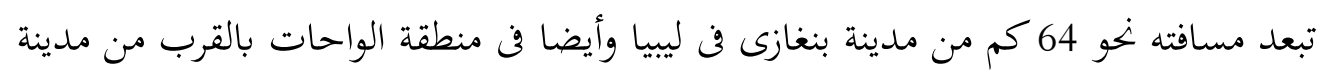
أوجلة وفى جبل الأخضر شرق ليبيا. تلك المواقع كانت هى مواقع الأحداث الحقيقية تقربيا. وانتهى فى 2 أوكتوبر 1979 وأول عرض عالمي للفيلم 4 أبريل 1981 فن دولة الكويت والولايات المتحدة الأمريكية ثم إلى أجزاء العالم. وقد اشترك في الفيلم مايقارب 250 مثثلا بالإضافة إلى إلى

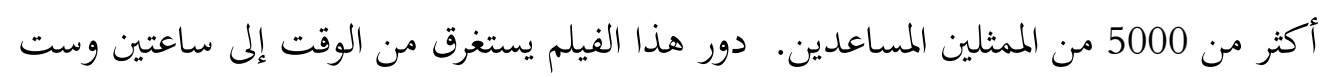

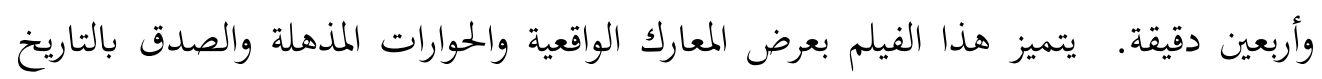
الإسلامى والعدل نخو المسلمين. لأن من عادة الفيلم الذي أصدره فنانو هوليوود ويتعلق بالإسلام, صوره بالتهمة غير الصالحة. فن سنة ألف وتسعمائة وإحدى عشرة تحت رئاسة بينيطو موسوليني وسيطرته، وسعت

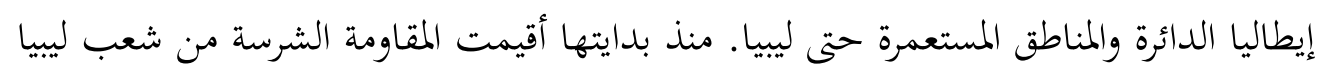
تحت قيادة عمر المختار. فعدم قدرة القادة الإيطاليين القدماء على وقف المقاومة التي قام بها عمر المختار وجيوشه يعتبر سببا لإرسال بينيطو موسوليني جنرال رودوفو غراتسياني ليكون وليا 


\section{Zaenal Masduqi}

على ليبيا مع الوظيفة العظمى وهي قبض عمر المختار وإهلاك مقاومة عسكريته. تدور تلك الأحداث الفيلمية في ليبيا عام 1929 حتى قبض عمر المختار في التاريخ 16 سبتمير 1931 وقام جنرال غراتسياني بالإعدام أمام شعبه في التاريخ 31 في نفس الشهر والسنة بعد خروج قرار المخكمة الإيطالية.

وابتدأ جنرال غراتسياني عملية الإهلاك نحو المقاومة المسلحة من تحريق البيوت وإفساد الزراعة وطرد سكاها إلى مكان خخصوص وسعي إلى قتله. ولكن عمر المختار قائد الحرب الذكى لـى ومستولى على الإستراتيجية السلاحية والميدانية في الصحراء. وأفاد معرفته عن جغرافيا ليبيا لنيل الفوز في كثير من الحروب ولاسيما أن الجنود الإيطاليين لم يستول بالدقة عن أحوال الصحراء.

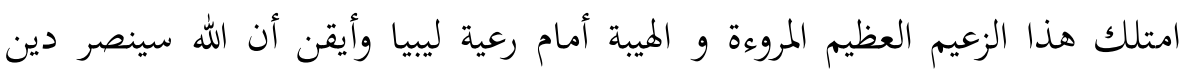

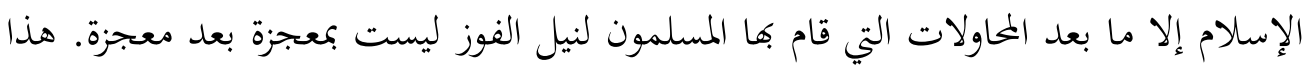

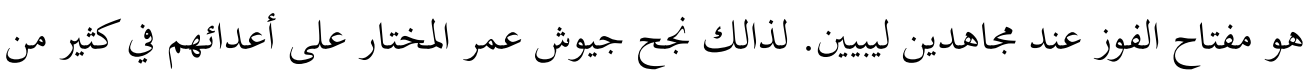
المعارك مهما كانت قيمة الجنود والأسلحة والقوة غير متكافئة وبعيدة جدا عن عندئن المستوي. علمنا أن هذا القائد العظيم يكافح بروحه وحماسته. إذا ضاعت هذه الحماسة منا

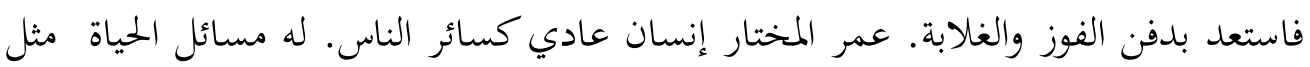
الحزن والخوف واليأس ولكنه يعرف كيف يقاومها ويحللها حتى تأتيهم المعونة من الله تعالى وإبدال المنى على اليأس. البيانات التاريخية

ولد عمر المختار في بطنا مدينة جنزور جبل الأحضر ليبيا من والديه الصالحين في سنة ألف وثمان مائة واثنتين وستين من الميلاد، وفي بعض المراجع ثمان وخمسين. واسم أبيه مختار بن عمر من عائلة فرحات ومن قبيلة منفة. (Ash-Shallabi, 2007)

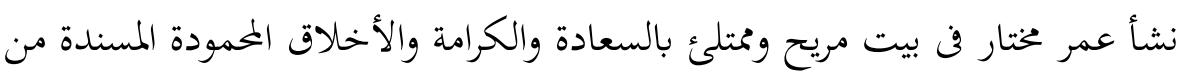
القرآن والحديث. توفي أبوه حينما قام بالحج وهو في صغر من عمره وشعر ذل الحياة كاليتيم. تعلم في مدرسة القرآن لقبيلته واستمرّ في مدرسة زغبوب في الفقه والحديث والتفسير. لقد ظهر

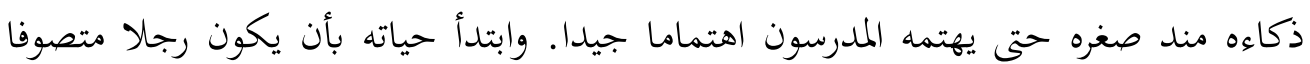




\section{Zaenal Masduqi}

وانتسب إلى الطريقة السانوسية ويخدم فيها حتى الموت اتبعه سكان ليبيا لا سيما بعد أن عرفوا دخول عمر المختار فيها. إزدادت القوة هذه الطريقة على المقاومة المسلحة باشتراك عمر فيها لأنه من المجاهدين القادرين على أن نشمرعن القوة الإيطالية. قدرته الدبلومسية والاتصالات الجيدة وسط قومهم يجعل الاتحاد بين القبائل الموجودة فن ليبيا قوية وأزودة للمقاومة وكانت القبائل قبل ذالك متفرقة ومتكسرة سبب ظهور الفتن من الإيطالية.

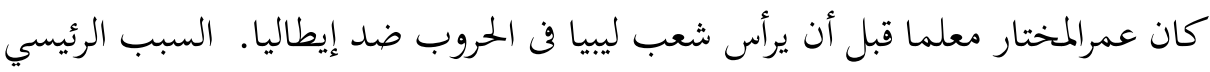
يجعله يقاوم الإحتلال الإيطالى حينما يرى الشعب الليبي يعانون لوجود المعاملة السيئة والاستبدادية من الجيوش الإيطالية, والغضب لسلوك بعض القيادة الصوفية السانوسية الذين عقدوالاستسلام والاستمتاع مع الاحتلال الإيطالي. بالألات الحربية البدائية وعلى ظهور الخيل,

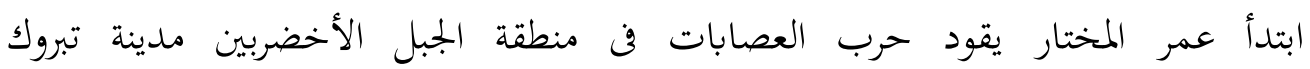
وبنغازي.(Tajudin, 2011)

تلك المنطقة صالحة جدا للغاية لتكون مركزا لحرب العصابات لكوها جبلية وكثيرة من المياه ومليئة بالأشجار. فن هذه المنطقة أيضا قبضه الجيوش الإيطالية في التاريخ 11سفتمبر 1931بعد عشرين عاما يتميز في الحروب التي تخسر القوات الإيطالية كثيرا. بنى هذه الطريقة الشيخ محمد على السانوسى فن سنة ألف وثمان مائة وسبع وثلاثين. الطريقة السانوسية ليست كالطريقة العادية كما كنا نعرف وإنما هى الحركة والتجديد والاجتهاد ومحاربة التقليد. بعيدة من الشرك والخرفات. لاتترك الدنيا بل يهتم بأمور الدنيا تحارب كثيرا أنواع الظلمات التى أمام عينيها (synopsis film the lion of the dessert,1981) انتشرت هذه الطريقة إلى أفريقيا الجنوبية وسودان و الصومال وبعض الدول العربية. هذه الطريقة تأثرت بالإمام أحمد إبن حنبل وأبي حامد الغزالى. وفي الدعوة استخدمت هذه الطدي الطريقة أساليب الحكمة والموعظة الحسنة وأكدت على المحاولات اليدوية والجهاد في سبيل الله بمعاندة المستعمرين الغربيين المحتلين على بلاد المسلمين. من المجال السياسى الإقليمى أنّ ليبيا حينذاك تكون قطعة من الولايات التابعة لخلافة الترك العثماني الواسعة التي تمتد ولاياتا إلى ثلاث قارات آسيا وأفريقيا وأوربا. ولكن في القرن 


\section{Zaenal Masduqi}

التاسع عشر إلى العشرين أصبحت هذه الخلافة ضعيفة وخضيعة بظهور القوة الجديدة من الدول

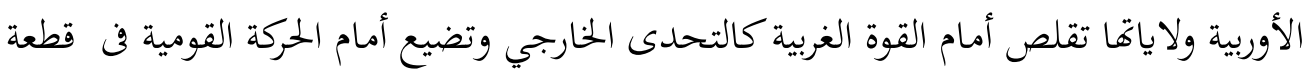
(Rogan, بلكان خصوصا كالتحدى الداخلي وفى مثل هذالحال لقبت بالمريض من أوربا.

سبب ظهور إيطاليا كمستعمرة على ليبيا هو ضعف الترك العثمانى ولإتساع الدوائر

المحتلة عند إيطاليين. سقطت ليبيا على يد إيطاليا فن سنة ألف وتسعمائة وإحدى عشرة ولم تهتم الخلافة لمساعدةًا الآ قليلا من الجنود التركية التى وضعت حول ليبيا. وفى مثل هذه الحالة قام عمر المختار بالمقاومة المسلحة قدر عشرين سنةعلى تنظيم الطريقة السانوسية. فرأت جيوش إيطاليا الأقوياء في مجال الأسلحة أن المجاهدين الليبيين فرقة مسلحة نئهة صغيرة, ولكن تحت قيادة عمر مختار تجعل قوة إيطاليا على حروب طويلة في الصحراء. ولكن التقدير لايمكن إنكاره الموت سيأتى. الأسلحة غير متوازنة يكفينا بحجة أن المجاهدين في مقده فيدار

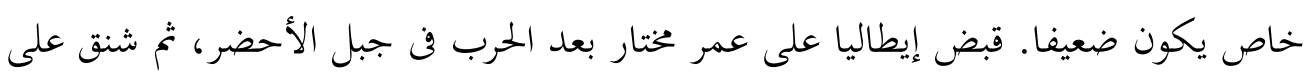
حبل المشنقة أمام رعيته ومتبعيه في يوم الأربعاء الموافق السادس عشر سنة إنس ألف وتسعمائة واحدة وثلاثين بعد الممارسة الجهادية سنوات عديدة. (Ash-Shallabi, 2007) تبسم هذا الشيخ العظيم قبل الإعدام شنقا لتناول الشهادة والرضا من الله عز وجل في 73 سنة من عمره. قال

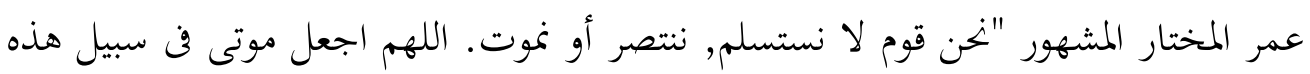
القضية المباركة." - n

$$
\text { البيانات التعبيرية }
$$

وفقا للتاريخ أن تشريع الجهاد على معنى القتال ليست دفعة واحدة على أعباء المسلمين

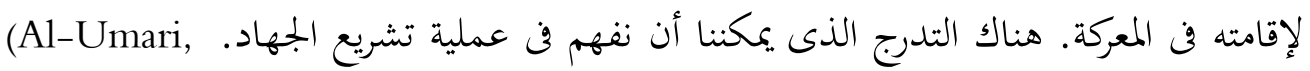
الأول طوال عصر مكة هناك الأمر على ألاّ يواجه Ardiana, Asro, \& Syadzili, 2003)

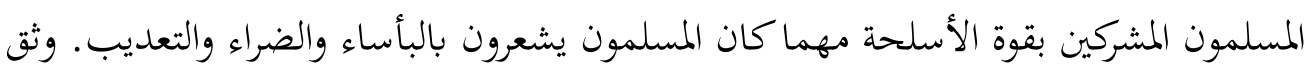
القرآن هذه الحالة في سورة النساء : 77 : "المُ تر إلى الذين قيل لهم كفّوا أيديكم وأقيمو الصلاة...". 


\section{Zaenal Masduqi}

هناك حكمة بالغة من هذا المنع وهى إرشاد المسلم على إصلاح نفسه وازدياد إيمانه

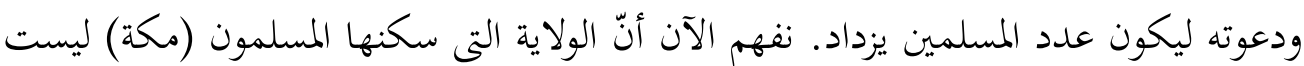
تحت رئاسة المسلمين وكانت جملتهم قليلة.

والثانى حينما هجر المسلمون إلى المدينة على دفاع الأنصار يمتلك المسلم على الولاية. حينئذ أمر بالجهاد لدفاع النفس والاعتقاد وقال الله تعالى أذن للذين يقاتلون بأفم ظلموا وإن الله على نصرهم لقدير (الحجر: 39). وقال أيضا وقاتلوا في سبيل الله الذين يقاتلونكم ولا تعتدوا

$$
\text { إن الله لا يحب المعتدين (البقرة: 190). }
$$

والثالث أمر الله المسلمين ليحاربوا المشركين وابتدؤوا بالحرب. هذه لسهولة نشر دعوة الإسلام أمام المشركين واستيلاء المسلمين على الدنيا. بهذه الطريقة لا أحد من المشركين محاربة المؤمنين في أمور دينهم أينما كانوا.

قال الله تعالى: "وقاتلوهم حتى لا تكون فتنة ويكون الدين كله لله فان انتهوا فإن الله بما

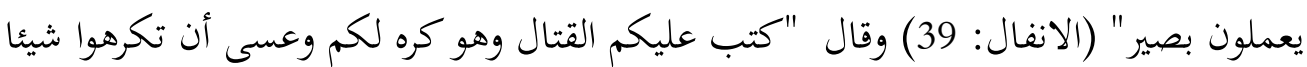
وهو خير لكم" (البقرة: 216) وقوله آخر :"قاتلو الذين لا يؤمنون بالله ولا باليوم الآخر" (التوبة:

عند رأى الباحث أن الجهاد فن أرض ليبيا في مرتبة تشريع الجهاد الثانية والثالثة لوجود التعذيبات والتدميرات والاحتلال الذى قام بها الإيطاليون لهؤلاء المسلمين في ليبيا. الأحوال الاجتماعية والسياسية التي كانت غير عادلة كمثل هذه تكون بينات واضحة لماذا ظهرت القوة الاجتماعية والسياسية والعسكرية على أساس الدين. ولابد من إقامة المقاومة

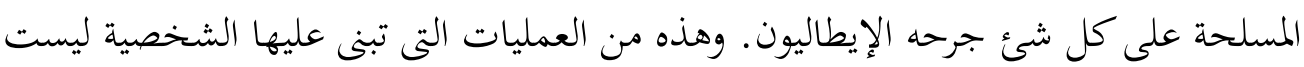
بالظواهر فقط وإنما تنتظم على منظمة جالية وسريعة. وامتلك عمر مختار منبع القوة على المقاومة لذالك جدير بالممارسة والتنظيم والتقرب إلى الله. (Prasetyo, 2002) هذذه كلها شهدت الدول العالمية قوة شعب ليبيا حين دمّره وقبضه وسجّنه الإيطاليون وقدر كتائب عمرالمختارعلى الدفاع عن كل هجوم ايطالي مهما كانوا من الأقليات. 
Zaenal Masduqi

أقوال عمر المختار في فيلم "أسد الصحراء" ومعانيها

1. "يأتون دائما كالأسد ويعودون كالمنعزم". هذا القول الجهادي الأول الذى حدث فن

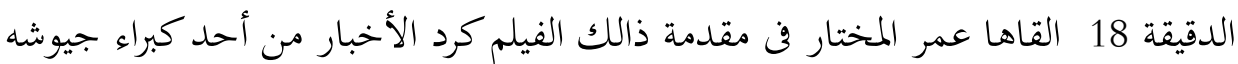

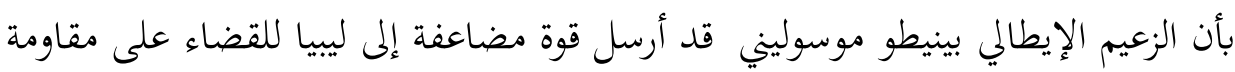

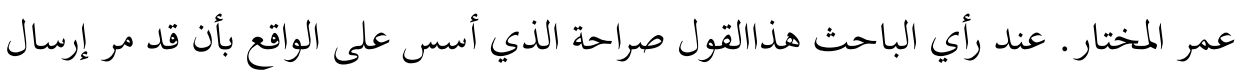

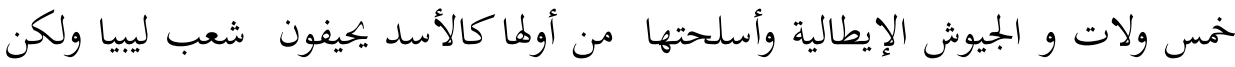
آخرهم كالمنعزم يموتون في ليبيا. هذالتعبير يمثل أن مجئ الجيوش الإيطالية بقوة قييمة ولكن الإلية

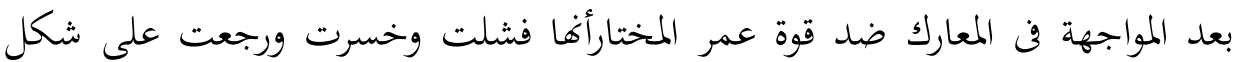
الهزيمة.

2. "قل لجنرالك ليس هنا مكانه". هذا لتعبير الجهادي الثاني الذي وجد الباحث في الدقيقة 45 من ذالك الفيلم بعد معركة صغيرة بأن القوة الإيطالية مغلوبة إلا رجلا واحدا. فتناول

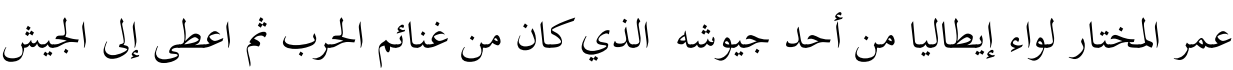

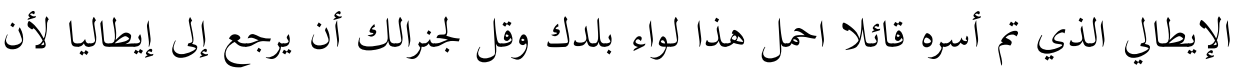

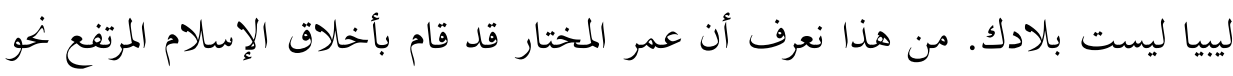

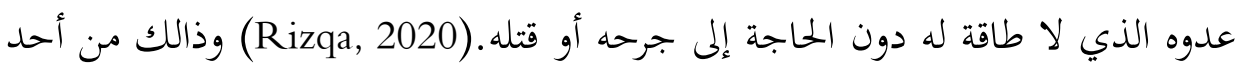
المشاهد السينمائية التى تجرإلى الدعوة بأن الإسلام دين السلام والرحمة إلى كافة الأنام.

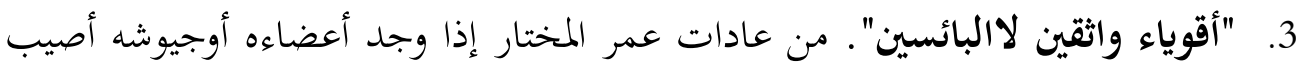

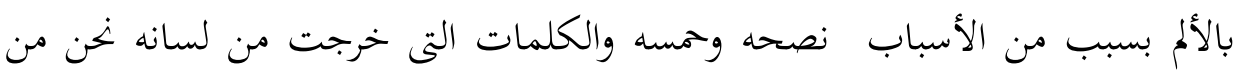

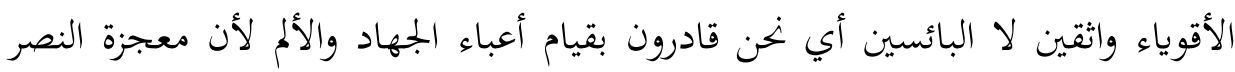

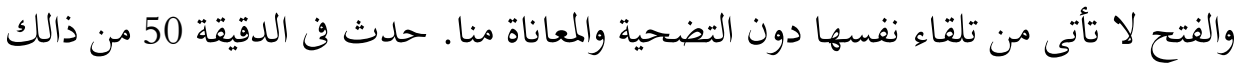

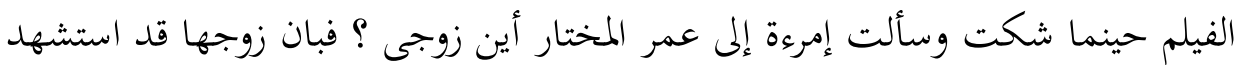

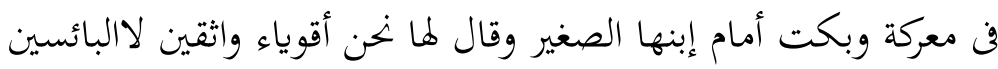

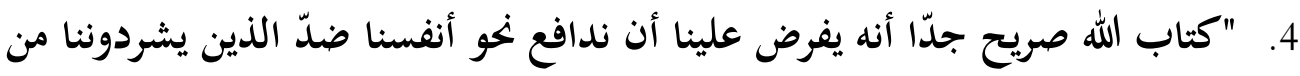
بيوتنا". هذا التعبير صريح جدا من لسان عمر المختار الموجود فن ذالك الفيلم أن نقيم 
Zaenal Masduqi

المقاومة السلاحية لمواجهة من كان يطردنا من بيوتنا أو بلادنا حتى يعطينا الله النصر أو

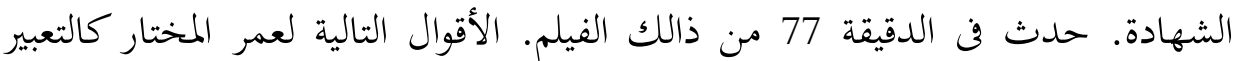
الجهادى على نفس المعنى وهو تحريض شعب ليبيا على الجهاد والمقاومة المسلاحة لمواجهة الاحتلال الإيطالي.

5. "مادمتم تحتلون أرضنا وما دمنا قادرين على الحرب يجب أن نحاربكم. كلما كان هناك استعمار قاسي ستكون هناك مقاومة بطولية من قبل المستعمرين وإن كانت بالأسلحة البدائية وبأقل قواة وجب على تنفيذها. لأن الالتزام بالمقاومة والقتال ضد الاحتلال

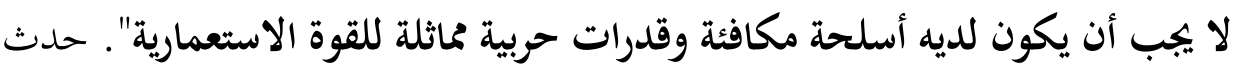
ذالك التعبير الجهادي في الدقيقة 68 من الفيلم عندما كانت هناك مفاوضة السلام بين قوات عمر المختار والمستعمرين الإيطاليين التى انتهت بعدم أي اتفاق. 6. "عندهم ثمانون مليون حرب وعندنا الله وهو الأهم كما أننا لانحرب وحدنا هناك إخوتنا الذين يحاربون على مختلف الجبل." حدث في الدقيقة 144من ذلك الفيلم عندما أعطى عمر المختار التوجيه وروح النضال على جيوشه في كهف مختبع قبل الحرب الكبرى, بعد

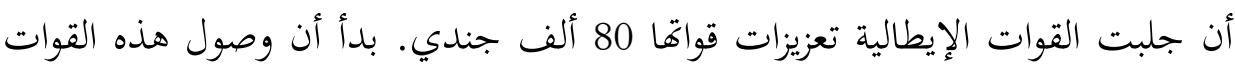
الإيطالية الضخمة قد أعجبت جيوش عمر المختار وقد يجرهم إلى الخوف. في هذه الحالة المخزنة خرج ذالك التعبير الجهادي الصريح جدا بكلمة المحاربة من لسان عمر المختار التى لئى إنى معناها المقاومة والمقاتلة. تلك القوات الإيطالية لن تخيف عمر المختار وجيوشه لأن عندهم الله المستعان.

7. "نحن باقون معكم إلى نهايتكم". حدث في الدقيقة 82 من ذالك الفيلم عندما فشلت قوة الاحتلال الإيطالي على طاولة المفاوضات لإقناع عمر المختار بالتخلى عن إغراء الحياة المريحة. أجاب عمر المختار فن آخر المفاوضة بقول نحن باقون معكم إلى فايتكم. هذ التعبير الجهادي بالغ للشعور لزيادة الروح والحماسة الداخلية والتنافسية في مواجهة القوى الاستعمارية, طالما بقيت القوة الاستعمارية في مكاها حتى انتهائها وضياعها. 
Zaenal Masduqi

\section{الحخلاصة والتوصيات}

بعد الملاحظة لمذا البحث القصير من خلال فيلم أسد الصحراء, وجد الباحث عن

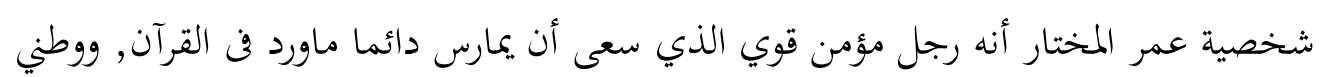

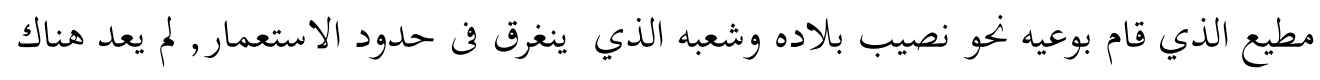

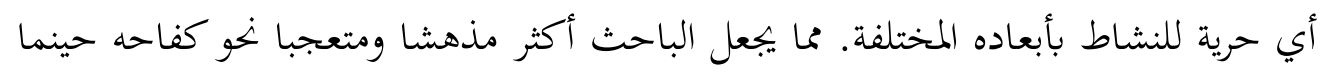
ذلك الوعي القومى الملفوف بالدين وتدينه الرائع ينتشر وينتقل إلى شعب ليبيا حتى أصبحت

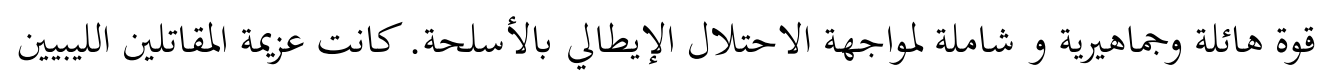
الذين قادهم عمر المختار للاستقلال بلادهم لعظيمة جدا, رغم أفم هزموا فن النهاية أمام

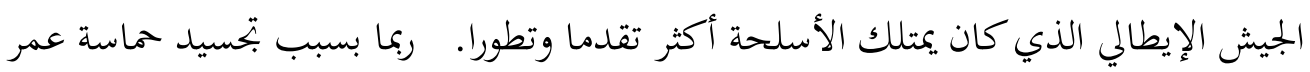
المختار في تثبيت الحالة النفسية لمقاتليه في جيشه من خلال التعبيرات الجهادية المذكورة أعلاه فعالا للغاية لفترة تصل إلى عشرين عاما. لايقتصر بهذا تعجب الباحث, وإنما أكثر من ذالك أصبح كفاحه وجهاده مصدر إلهام وموطئ قدم لمناطق أخرى من العالم الإسلامى. بناءعلى التاريخ, كانت فترة جهاد عمر المختار

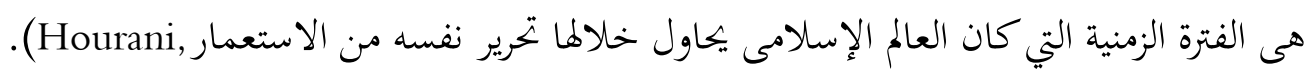

إن محاولة التحرر الذاتى من الاستعمار القائم على القومية هي حطة واقعية، يجب اتخادها

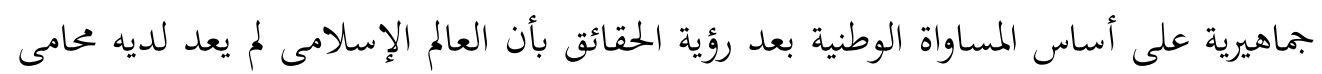
دولى قوي بعد تصفية الخلافة العثمانية الإسلامية في التاريخ 4 مارس 1924. منذ ذالك اليوم أصبحت القومية نظام الدولة على سائر العالم الإسلامى بعد الإرادة و القدرة على إعادة بناء نظام الخلافة غير متوفرة وناجحة. (Pandawa, 2021)

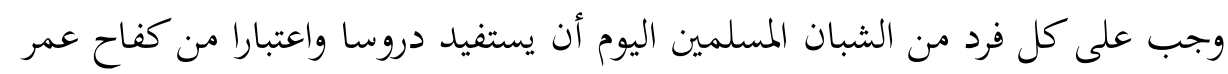

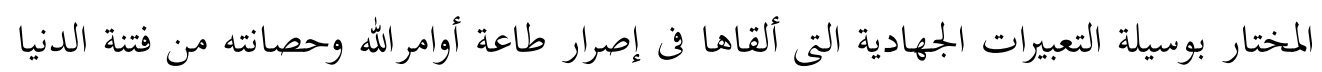
التي ستضعف جهاده وكرامته. والآن قد مات عمر المختار قدر تسعين سنة الماضية ولكن إسمه 


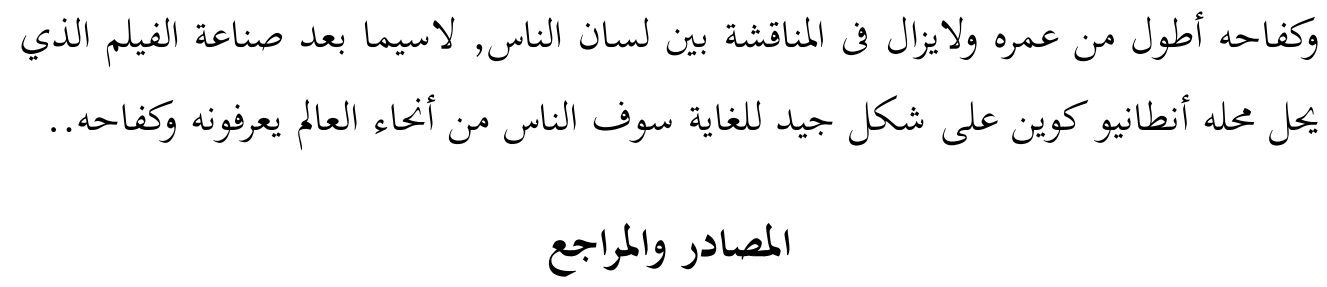

Al-Umari, A. D., Ardiana, A. H., Asro, H., \& Syadzili, A. F. (2003). Tolak Ukur Peradaban Islam : Arkeologi Sejarah Madinah dalam Wacana Trans-Global. Yogyakarta: Ircisod.

Ash-Shallabi, A. (2007). Umar Mukhtar Napak Tilas Jihad Sang Singa Padang Pasir (S. M. A. Hasibuan, trans.). Solo: Jazera Pustaka Salam.

Hourani, A. (1991). Sejarah Bangsa-Bangsa Muslim (I. A. Bakar, trans.). Bandung: Mizan Pustaka.

Pandawa, N. (2021). Khilafah dan Ketakutan Belanda Riwayat PanIslamisme dari Istanbul sampai Batavia, 1882-1928. Bogor: Komunitas Literasi Islam.

Rizki, S., \& Golubović, J. (2020). An Analysis of speech act of Omar Mukhtar's utterances in Lion of the Dessert movie. Englisia Journal of Language and Humanities, 7(2), 195-210. https:/ / doi.org/https:/ / doi.org/10.22373/ej.v7i2.6358

Rizqa, H. (2020). Inspirasi Perjuangan Antikolonial Umar al-Mukhtar. Retrieved 24 March 2021, from republika.id website: https:// www.republika.id/posts/14374/inspirasi-perjuanganantikolonial-umar-al-mukhtar

Rogan, E. (2017). The Fall of Khilafah: Perang Besar Yang Meruntuhkan Khilafah Utsmaniyah Dan Mengubah Selamanya Wajah Timur Tengah (F. Yamani, Trans.). Jakarta: Serambi Ilmu Semesta. 
Zaenal Masduqi

Rosyid, M. F., Isbah, F., \& Assegaf, A. (2020). Al-Lughatul Al-'Arabiyah Kamabda'i Al-Tarbawi Al-Diniy. IJAS: Indonesian Journal of Arabic Studies, 2(2), 107-120.

Sobur, A. (2001). Analisis Teks Media-Suatu Pengantar Untuk Analisis Wacana, Analisis Semiotik, dan Analisis Framing. Bandung: PT. Remaja Rosdakarya.

Tajudin, Q. (2011). Reportase dari Libya: Pemberontak Terinspirasi Omar Mukhtar. Retrieved 2 March 2021, from dunia.tempo.co website: https://dunia.tempo.co/read/326222/reportase-darilibya-pemberontak-terinspirasi-omar-mukhtar $/$ full\&view $=$ ok 
Zaenal Masduqi

This Page Is Intentionally Left Blank

تركت هذه الصفحة فارغة عمدا

'Halaman Ini Sengaja Dikosongkan' 